TRANSACTIONS OF THE

AMERICAN MATHEMATICAL SOCIETY

Volume 337, Number 1, May 1993

\title{
THE MARTIN BOUNDARY IN NON-LIPSCHITZ DOMAINS
}

\author{
RICHARD F. BASS AND KRZYSZTOF BURDZY
}

\begin{abstract}
The Martin boundary with respect to the Laplacian and with respect to uniformly elliptic operators in divergence form can be identified with the Euclidean boundary in $C^{\gamma}$ domains, where
\end{abstract}

$$
\gamma(x)=b x \log \log (1 / x) / \log \log \log (1 / x),
$$

$b$ small. A counterexample shows that this result is very nearly sharp.

\section{INTRODUCTION}

In the past few years a number of results that were previously known for Lipschitz domains have been shown to hold in much wider classes of domains. Among these, for example, are the mutual absolute continuity of harmonic measure and surface measure (in some $L_{1}^{p}$ domains) [JK2], the boundary Harnack principle (in all Hölder domains) [BB2, BBB], the parabolic boundary Harnack principle (in some $L^{p}$ domains) [BB3], intrinsic ultracontractivity (in uniformly Hölder domains) [Bñ], and upper bounds for the heat kernel with Neumann boundary conditions (in all Hölder domains) [BH].

In this paper we consider the problem of the identification of the Martin boundary. Here the situation is quite a bit more delicate. We prove

Theorem 1.1. Both the Martin boundary and the minimal Martin boundary are equal to the Euclidean boundary in bounded $C^{\gamma}$ domains for

$$
\gamma(x)=b x \frac{\log \log (1 / x)}{\log \log \log (1 / x)}
$$

provided $b$ is sufficiently small.

For definitions of Martin boundary, minimal Martin boundary, and $C^{\gamma}$ domains, see $\S 2$. Roughly, however, a $C^{\gamma}$ domain is one where the boundary can be represented locally as the graph of a function whose modulus of continuity is no worse than $\gamma$. Much better results can be obtained in $\mathbb{R}^{2}$ by complex analytic techniques, so throughout this paper we restrict attention to $\mathbb{R}^{d}, d \geq 3$. Our results are also valid for the Martin boundary with respect to uniformly elliptic operators in divergence form with bounded and measurable coefficients.

Received by the editors February 13, 1991.

1980 Mathematics Subject Classification (1985 Revision). Primary 31C35; Secondary 60J50.

Key words and phrases. Martin boundary, Martin kernel, harmonic functions, minimal harmonic, divergence form operators, conditioned Brownian motion, $h$-processes.

Research partially supported by NSF grants DMS 88-22053 and DMS 89-01255. 
Our results are very nearly sharp. We also prove

Theorem 1.2. For each $b>0$ there exists a bounded $C^{\gamma}$ domain in $\mathbb{R}^{3}$ with

$$
\gamma(x)=b x \log \log (1 / x)
$$

for which the Martin boundary is different from the Euclidean boundary.

The Martin boundary was introduced in [M] in order to give a representation of positive harmonic functions in a domain akin to the Poisson integral formula for a ball. The identification of the Martin boundary with the Euclidean boundary for Lipschitz domains was first given in [HW]. Alternate proofs have been given by [JK1 and $\mathrm{BB} 1]$. In [JK3] Jerison and Kenig showed that the Martin boundary equals the Euclidean boundary in nontangentially accessible domains. Although nontangentially accessible domains share many of the scaling and potential-theoretic properties of Lipschitz domains, their boundaries can be quite wild. Ancona has a number of papers on Martin boundary; see [A] and the references therein.

In $\S 2$ we prove Theorem 1.1: we prove the equality of the Martin boundary with the Euclidean boundary in bounded $C^{\gamma}$ domains, $\gamma(x)$ as in (1.1) and we prove that the Martin kernel associated with each Euclidean point is a minimal harmonic function. In $\S 3$ we give the necessary lemmas that allow us to extend the results of $\S 2$ to divergence form operators. And in $\S 4$ we prove Theorem 1.2 .

Let us give a heuristic explanation for why the critical modulus of continuity for our problem is close to $x \log \log (1 / x)$. We start with a domain $D_{1}$ which is the upper halfspace from which two hemispheres were removed, i.e.,

$D_{1}=\left\{x^{1}>0\right\}-(\{|x-(0,1,0, \ldots, 0)| \leq 1\} \cup\{|x-(0,-1,0, \ldots, 0)| \leq 1\})$.

Martin [M] proved that the Euclidean point 0 corresponds in $D_{1}$ to infinitely many Martin boundary points.

Now modify $D_{1}$ to obtain a domain $D$ which has the same general shape as $D_{1}$ but lies above the graph of a function with modulus of continuity $x \log \log (1 / x)$. The part of $\partial D$ whose distance from 0 is between $2^{-k-1}$ and $2^{-k}$ consists mostly of two almost flat annuli; the distance $a_{k}$ between them satisfies $a_{k} \log \log \left(1 / a_{k}\right) \approx 2^{-k}$ from which we obtain $a_{k} \approx 2^{-k} / \log k$. The ratio of the length to width of the canal between these two annuli is $\approx 2^{-k} /\left(2^{-k} / \log k\right)=\log k$. The chances that Brownian motion conditioned to go to 0 will go from one side of the canal to the other are of order $\exp (-\log k)=$ $1 / k$. The series $\sum 1 / k$ is divergent, so by the Borel-Cantelli lemma the process will change the direction of approach infinitely often and there is only one Martin boundary point corresponding to the approach of Brownian motion to 0 . A slight perturbation of the domain will make the series summable and the process will change its direction of approach only finitely many times. In this case, we will obtain, by symmetry, at least 2 distinct Martin boundary points, corresponding to Brownian motion approaching 0 from two sides.

The letter $c$ with subscripts will denote constants with values in $(0, \infty)$ which may change from proposition to proposition but do not change within a proposition and its proof. For details on the Martin boundary see Doob [Do]. For more information on the relationship between Brownian motion and harmonic functions, see [Do and PS]. 


\section{MARTIN BOUNDARY}

We begin with some notation. Suppose $d \geq 3$. The Euclidean closure of $A \subset \mathbb{R}^{d}$ will be denoted $\bar{A}$. Write $x=\left(x^{1}, \ldots, x^{d}\right)=\left(\tilde{x}, x^{d}\right)$, where $\tilde{x}=$ $\left(x^{1}, \ldots, x^{d-1}\right)$. Suppose $\gamma:[0, \infty) \rightarrow[0, \infty)$. We let $C^{\gamma}$ be the set of all functions $f: \mathbb{R}^{d-1} \rightarrow \mathbb{R}$ such that $|f(x)-f(y)| \leq \sup _{0<r \leq|x-y|} \gamma(r)$ for all $x, y$ $\in \mathbb{R}^{d-1}$. We say $D$ is a $C^{\gamma}$ domain if for all $x \in D$ there exist a neighborhood $U_{x}$ of $x$, an orthonormal coordinate system $C S_{x}$, and a $C^{\gamma}$ function $\Gamma_{x}$ such that

Define

$$
D \cap U_{x}=\left\{y: y=\left(\tilde{y}, y^{d}\right) \text { in } C S_{x}, y^{d}>\Gamma_{x}(\tilde{y})\right\} \cap U_{x}
$$

$$
L_{2}(x)=\log \log (1 / x), \quad \varphi(x)=\frac{\log \log (1 / x)}{\log \log \log (1 / x)}
$$

if $x \in\left(0, \exp \left(-e^{e}\right)\right), e$ otherwise.

We take $b>0$, the value of $b$ to be chosen later. Suppose $\Gamma: \mathbb{R}^{d-1} \rightarrow \mathbb{R}$ is in $C^{b x \varphi(x)}$, and for now suppose $D=\left\{x \in \mathbb{R}^{d}: x^{d}>\Gamma(\tilde{x})\right\}$. Let $d(x)=$ $\operatorname{dist}(x, \partial D), \delta(x)=x^{d}-\Gamma(\tilde{x})$, and $B(x, r)=\left\{y \in \mathbb{R}^{d}:|y-x|<r\right\}$.

Let

$$
\begin{aligned}
\Delta(x, a, r) & =\left\{y \in D: \Gamma(\tilde{y})<y^{d}<\Gamma(\tilde{y})+a,|\tilde{y}-\tilde{x}|<r\right\}, \\
\partial^{u} \Delta(x, a, r) & =\left\{y \in \partial \Delta(x, a, r): y^{d}=\Gamma(\tilde{y})+a\right\} \quad \text { (“u” for upper), }
\end{aligned}
$$

and

$$
\partial^{s} \Delta(x, a, r)=\{y \in \partial \Delta(x, a, r):|\tilde{y}-\tilde{x}|=r\} \quad \text { ("s" for side). }
$$

Let $\left(X_{t}, P^{x}\right)$ be standard Brownian motion in $\mathbb{R}^{d}$. For any Borel set $A$, let

$$
T(A)=\inf \left\{t>0: X_{t} \in A\right\} .
$$

Before plunging into the proof of Theorem 1.1, let us give a brief overview. To show that no Euclidean point corresponds to more than one Martin boundary point, it suffices to fix $x, x_{0} \in D$ and to show $g(x, y) / g\left(x_{0}, y\right)$ is uniformly continuous for $y$ near the boundary, where $g$ is the Green function for the domain $D$. We do this by showing uniform continuity for $g(x, y) / h(y)$ and $g\left(x_{0}, y\right) / h(y)$, where $h(y)$ (given in $\left.(2.5)\right)$ is the probability of exiting a certain "box" $\Delta(0, \psi(a), \psi(a))$ from its upper side. Write $u(y)$ for $g(x, y)$. Consider two boxes $W_{k+1} \subset W_{k}$. By the usual Harnack inequality, the values of $u$ along the upper side of $W_{k}$ are all comparable, and similarly for $h$. By the techniques of [BB2] (see Lemma 2.4), the probability that the $h$-path transform of Brownian motion starting in $W_{k+1}$ will exit $W_{k}$ through its upper boundary can be bounded below. We conclude that the oscillation of $u / h$ on $W_{k+1}$ must be less than $\rho_{k}$ times the oscillation of $u / h$ on $W_{k}$ for suitable $\rho_{k}$. Taking a decreasing sequence $W_{k}$ and showing $\prod \rho_{k}=0$ gives the uniform continuity of $u / h$.

The following lemma is Lemma 2.2 of [BB2].

Lemma 2.1. There exists $c_{1} \in(0,1)$ such that if $k>0, a>0, r \geq a k$, and $y \in \Delta(x, a, r)$ with $\tilde{y}=\tilde{x}$, then

$$
P^{y}\left(X_{T(\partial \Delta(x, a, r))} \in \partial^{s} \Delta(x, a, r)\right) \leq c_{1}^{k} .
$$


Lemma 2.2. (cf. Lemma 2.3 of [BB2]) For each $b \in(0,1)$ there exists $a_{0}=$ $a_{0}(b)$ and $c_{1}$ (independent of $b$ ) such that if $a<a_{0}$ and $\delta(y)=a$, then

(a) if $r \geq 4 a, P^{y}\left(X_{T(\partial \Delta(y, 2 a, r))} \in \partial^{u} \Delta(y, 2 a, r)\right) \geq \exp \left(-c_{1} b \varphi(a)\right)$;

(b) if $r \geq 2^{k+1} a$,

$$
P^{y}\left(X_{T\left(\partial \Delta\left(y, 2^{k} a, r\right)\right)} \in \partial^{u} \Delta\left(y, 2^{k} a, r\right)\right) \geq \exp \left(-c_{1} b \sum_{i=1}^{k} \varphi\left(2^{i-1} a\right)\right) .
$$

Proof. Since $\Gamma \in C^{b x \varphi(x)}$, we observe that $d(y) \geq c_{2} a / b \varphi(a)$. Let $y_{i}=$ $\left(\tilde{y}, y^{d}+i c_{2} a / 2 b \varphi(a)\right), B_{i}=B\left(y_{i}, c_{2} a / b \varphi(a)\right), \quad i=0,1, \ldots, n$, where $n=$ $\left[4 b \varphi(a) / c_{2}\right]+3$. Since $D$ is the region above $\Gamma, d\left(y_{i}\right)$ is increasing, and each $B_{i} \subset D$.

Let $B=\bigcup_{i=1}^{n} B_{i}$. Let $h$ be the harmonic function on $B$ with boundary values 1 on $\partial B \cap \partial B_{n}$, and 0 on the remainder of $\partial B$. By standard properties of Brownian motion, $h\left(y_{n}\right) \geq c_{3}$. By Harnack's inequality in $B_{i}, h\left(y_{i}\right) \geq$ $c_{4} h\left(y_{i+1}\right)$. So by an induction argument,

$$
h(y)=h\left(y_{0}\right) \geq c_{3}\left(c_{4}\right)^{n}=c_{3} \exp \left(-n \log \left(c_{4}^{-1}\right)\right) \geq \exp \left(-c_{1} b \varphi(a)\right),
$$

using $a<a_{0}$. By our choice of $n$, we see that the desired probability in (a) is bounded below by $P^{y}\left(X_{T(\partial B)} \in \partial B_{n}\right)=h(y)$.

If we let $S_{i}=\inf \left\{t: X_{t} \in \partial^{u} \Delta\left(y, 2^{i} a, 2 \cdot 2^{i} a\right)\right\}$, then (b) follows from (a) by the strong Markov property applied at the times $S_{i}$ and an induction argument.

Lemma 2.3. Suppose $v$ is nonnegative and harmonic in $\Delta\left(y, 2^{k+1} a, 2^{k+2} a\right)$, $\delta(y)=a$, and $z=\left(\tilde{y}, y^{d}+2^{k} a\right)$. Then

$$
v(y) \geq \exp \left(-c_{1} b k \varphi(a)\right) v(z), \quad v(z) \geq \exp \left(-c_{1} b k \varphi(a)\right) v(y) .
$$

Proof. Let $y_{1}=\left(\tilde{y}, y^{d}+c_{2} n a / 2 b \varphi(a)\right)$, where $c_{2}$ and $n$ are as in the proof of Lemma 2.2. Constructing the chain of balls $B_{i}$ connecting $y$ and $y_{1}$ as in that proof, we see that

$$
v(y) \geq \exp \left(-c_{1} b \varphi(a)\right) v(z), \quad v(z) \geq \exp \left(-c_{1} b \varphi(a)\right) v(y)
$$

by Harnack's inequality. This proves the corollary in the case $k=1$.

An induction argument together with the fact that

$$
\exp \left(-c_{1} b \sum_{i=1}^{k} \varphi\left(2^{i} a\right)\right) \geq \exp \left(-c_{2} b k \varphi(a)\right)
$$

for $a<a_{0}$ gives the general case.

Lemma 2.4. For each $b \in(0,1)$ there exist $a_{0}=a_{0}(b)$ and $K_{1}$ and $c_{1}$ (independent of $b)$ such that if $a \in\left(0, a_{0}\right), R \geq K_{1} b a \varphi(a)$,

$$
\begin{aligned}
& H_{1}=\left\{X_{T(\partial \Delta(0,2 a, 2 R))} \in \partial^{s} \Delta(0,2 a, 2 R)\right\} \quad \text { and } \\
& H_{2}=\left\{X_{T(\partial \Delta(0,2 a, 2 R))} \in \partial^{u} \Delta(0,2 a, 2 R)\right\},
\end{aligned}
$$

then

$$
P^{x}\left(H_{1}\right) \leq c_{1} P^{x}\left(H_{2}\right) \text { for all } x \in \partial \Delta(0, a, R) \text {. }
$$


Proof. We follow the proof of Theorem 2.4 of [BB2] very closely. Write $\beta_{k}=$ $2^{-k}, r_{k}=2 R-(R / 8) \sum_{i=0}^{k}(1+i)^{-2}$. Note $r_{0}=15 R / 8$ and $\inf _{k} r_{k}>R$. Take $a_{0}$ small enough so that $b \varphi\left(a_{0}\right) \geq 1$.

Let

$$
J_{k}=\left\{y \in D: y^{d} \in\left[\Gamma(\tilde{y})+a \beta_{k+1}, \Gamma(\tilde{y})+a \beta_{k}\right],|\tilde{y}| \leq r_{k}\right\}, \quad k=0,1, \ldots
$$

By Lemma 2.2(b), if $z \in J_{0}$, then $P^{z}\left(H_{2}\right) \geq \exp \left(-c_{3} b \varphi(a)\right), c_{3}$ independent of $a$. On the other hand, if $z \in J_{0}$, then by Lemma 2.1, $P^{z}\left(H_{1}\right) \leq$ $\exp \left(-c_{4} K_{1} b \varphi(a)\right)$. So by taking $K_{1}$ large enough (independent of $\left.a_{0}\right), P^{z}\left(H_{1}\right)$ $\leq P^{z}\left(H_{2}\right)$ for $z \in J_{0}$.

Let $d_{m}=\sup _{z \in J_{m}} P^{z}\left(H_{1}\right) / P^{z}\left(H_{2}\right)$. From what we have just observed, $d_{0} \leq$ 1. Since $\Delta(0, a, R) \subset \bigcup_{k=0}^{\infty} J_{k}$, to prove the theorem it suffices to prove $\sup _{m} d_{m}<\infty$.

Fix $m$ and suppose $z \in J_{m+1}$. For the remainder of the proof, write

$$
\Delta_{m}=\Delta\left(z, a \beta_{m+1}, r_{m}-r_{m+1}\right), \quad U_{m}=T\left(\partial \Delta_{m}\right) .
$$

By the strong Markov property,

$$
P^{z}\left(H_{1}\right) \leq E^{z}\left(P^{X_{U_{m}}}\left(H_{1}\right) ; X_{U_{m}} \in \partial^{u} \Delta_{m}\right)+P^{z}\left(X_{U_{m}} \in \partial^{s} \Delta_{m}\right)
$$

and

$$
P^{z}\left(H_{2}\right) \geq E^{z}\left(P^{X_{U_{m}}}\left(H_{2}\right) ; X_{U_{m}} \in \partial^{u} \Delta_{m}\right) .
$$

Since $\partial^{u} \Delta_{m} \subseteq J_{m}$ when $z \in J_{m+1}$, using (2.4) we see that the first term on the right-hand side of $(2.3)$ is bounded by

$$
d_{m} E^{z}\left(P^{X_{U_{m}}}\left(H_{2}\right) ; X_{U_{m}} \in \partial^{u} \Delta_{m}\right) \leq d_{m} P^{z}\left(H_{2}\right) .
$$

By Lemma 2.1 the second term on the right of (2.3) is bounded above by

$$
\exp \left(-c_{4} / a(m+2)^{2} \beta_{m+1}\right) \leq \exp \left(-c_{4} b K_{1} \varphi(a) / m^{2} \beta_{m+1}\right)
$$

On the other hand, by Lemma $2.2(\mathrm{~b}), P^{z}\left(H_{2}\right)$ is bounded below by

$$
c_{5} \exp \left(-c_{3} b \sum_{i=-m-2}^{0} \varphi\left(2^{i} a\right)\right) \geq c_{5} \exp \left(-c_{6} b m^{3} \varphi(a)\right)
$$

Take $K_{1}$ larger if necessary so that $2 c_{6} m^{3} \leq c_{4} K_{1} / m^{2} \beta_{m+1}$ for all $m \geq 1$. We can choose $c_{7}$ such that for all $m$

$$
c_{5} \exp \left(-c_{6} b m^{3} \varphi(a)\right) \geq c_{7}^{-1} m^{2} \exp \left(-c_{4} b K_{1} \varphi(a) / m^{2} \beta_{m+1}\right) .
$$

So for $a<a_{0}$

$$
\begin{aligned}
P^{z}\left(H_{1}\right) & \leq d_{m} P^{z}\left(H_{2}\right)+\exp \left(-c_{4} b K_{1} \varphi(a) / m^{2} \beta_{m+1}\right) \\
& \leq d_{m} P^{z}\left(H_{2}\right)+c_{7} m^{-2} c_{5} \exp \left(-c_{6} b m^{3} \varphi(a)\right) \\
& \leq d_{m} P^{z}\left(H_{2}\right)+c_{7} m^{-2} P^{z}\left(H_{2}\right)
\end{aligned}
$$

Hence

$$
d_{m+1} \leq d_{m}+c_{7} m^{-2}
$$

or $\sup _{m} d_{m} \leq d_{0}+c_{7} \sum_{m=1}^{\infty} m^{-2} \stackrel{\mathrm{df}}{=} c_{1}<\infty$, as required.

Define

$$
\psi(a)=2 K_{1} b a(\varphi(a))^{5}
$$


Lemma 2.5. For each $b \in(0,1)$ there exists $a_{0}=a_{0}(b)$ and $c_{1}$ (independent of $b)$ such that if $a<a_{0}, v$ is nonnegative and harmonic in $\Delta(0, \psi(a), \psi(a))$, $|\tilde{x}-\tilde{y}| \leq 16 K_{1} b a \varphi(a)$, and $\delta(x)=\delta(y)=a$, then

$$
v(y) \geq \exp \left(-c_{1} b L_{2}(a)\right) v(x) .
$$

Proof. Let $w=\left(\tilde{x}, x^{d}+a(\varphi(a))^{4}\right), z=\left(\tilde{y}, y^{d}+a(\varphi(a))^{4}\right)$. Since $\Gamma \in C^{b x \varphi(x)}$, we have

$$
d(w) \geq \delta(w) / c_{2} b \varphi(\delta(w)) \geq \delta(w) / c_{2} b \varphi(a)>c_{3} a(\varphi(a))^{3} / b
$$

if $a_{0}$ is small enough, and similarly for $d(z)$. Since $b<1$, note also that $|z-w| \leq c_{4} a(\varphi(a))^{2}$. Provided $a_{0}$ is small enough, we can use Harnack's inequality in the ball $B\left(w, 2 c_{4} a(\varphi(a))^{2}\right)$ to get $v(z) \geq c_{5} v(w)$.

By Lemma 2.3 we have

$$
\begin{aligned}
v(y) & \geq \exp \left(-c_{6} b \varphi(a) \log (\varphi(a))^{4}\right) v(z), \\
v(w) & \geq \exp \left(-c_{6} b \varphi(a) \log (\varphi(a))^{4}\right) v(x) .
\end{aligned}
$$

To complete the proof note that $\varphi(a) \log (\varphi(a))^{4} \leq c_{7} L_{2}(a)$ for $a<a_{0}$ if $a_{0}$ is sufficiently small.

Now define

$$
h(x)=P^{x}\left(X_{T(\partial \Delta(0, \psi(a), \psi(a)))} \in \partial^{u} \Delta(0, \psi(a), \psi(a))\right) .
$$

Of course, $h$ is positive and harmonic in $\Delta(0, \psi(a), \psi(a))$. Let $\left(X_{t}, P_{h}^{x}\right)$ be the $h$-path transform of Brownian motion in $\Delta(0, \psi(a), \psi(a))$, i.e., Brownian motion conditioned by $h$; see [Do] for details.

Lemma 2.6. For each $b \in(0,1)$ there exist $a_{0}=a_{0}(b)$ and $c_{1}, c_{2}$ (independent of $b)$ such that if $a<a_{0}, K_{1} b a \varphi(a) \leq R \leq 2 K_{1} b a \varphi(a), H_{2}$ is defined as in (2.2), and $x \in \Delta(0, a, R)$, then

$$
P_{h}^{x}\left(H_{2}\right) \geq c_{1} \exp \left(-c_{2} b L_{2}(a)\right) .
$$

Proof. Note $h$ has boundary values 0 on $\partial^{s} \Delta(0, \psi(a), \psi(a))$, hence $\partial h / \partial x^{d}$ $=0$ there. Since $h \leq 1$ and $h$ has boundary values 1 on $\partial^{u} \Delta(0, \psi(a), \psi(a))$, then $\partial h / \partial x^{d} \geq 0$ on the upper boundary. Finally, since $h \geq 0$ and $h=0$ on $\partial D$, then $\partial h / \partial x^{d} \geq 0$ on $\partial D \cap \partial \Delta(0, \psi(a), \psi(a))$. By the maximum principle, then, $\partial h / \partial x^{d} \geq 0$ in $\Delta(0, \psi(a), \psi(a))$.

Write $\Delta$ for $\Delta(0,2 a, 2 R)$. Since $h$ is harmonic,

$$
\begin{aligned}
h(x) & =E^{x}\left(h\left(X_{T(\partial \Delta)}\right) ; X_{T(\partial \Delta)} \in \partial^{u} \Delta\right)+E^{x}\left(h\left(X_{T(\partial \Delta)}\right) ; X_{T(\partial \Delta)} \in \partial^{s} \Delta\right) \\
& \leq E^{x}\left(h\left(X_{T(\partial \Delta)}\right) ; X_{T(\partial \Delta)} \in \partial^{u} \Delta\right)+\left(\sup _{\partial^{s} \Delta} h\right) P^{x}\left(X_{T(\partial \Delta)} \in \partial^{s} \Delta\right) .
\end{aligned}
$$

By Lemma 2.4, $P^{x}\left(X_{T(\partial \Delta)} \in \partial^{s} \Delta\right) \leq c_{3} P^{x}\left(X_{T(\partial \Delta)} \in \partial^{u} \Delta\right)$. Since $\partial h / \partial x^{d} \geq 0$, $h$ is nondecreasing as a function of $x^{d}$, so $\sup _{\partial^{s} \Delta} h \leq \sup _{\partial^{u} \Delta} h$. By Lemma 2.5, $\sup _{\partial^{u} \Delta} h \leq \exp \left(c_{4} b L_{2}(a)\right) \inf _{\partial^{u} \Delta} h$. Therefore

$$
\begin{aligned}
h(x) \leq & E^{x}\left(h\left(X_{T(\partial \Delta)}\right) ; X_{T(\partial \Delta)} \in \partial^{u} \Delta\right) \\
& +c_{3} \exp \left(c_{4} b L_{2}(a)\right)\left(\inf _{\partial^{u} u} h\right) P^{x}\left(X_{T(\partial \Delta)} \in \partial^{u} \Delta\right) \\
\leq & \left(1+c_{3} \exp \left(c_{4} b L_{2}(a)\right) E^{x}\left(h\left(X_{T(\partial \Delta)}\right) ; X_{T(\partial \Delta)} \in \partial^{u} \Delta\right) .\right.
\end{aligned}
$$


Hence

$$
P_{h}^{x}\left(H_{2}\right)=\frac{E^{x}\left(h\left(X_{T(\partial \Delta)}\right) ; X_{T(\partial \Delta)} \in \partial^{u} \Delta\right)}{h(x)} \geq c_{5} \exp \left(-c_{4} b L_{2}(a)\right) .
$$

Remark 2.7. Since we know the boundary Harnack principle holds for Hölder domains [BBB], it holds for the domain $D$ that we have been considering, as well as any $C^{b x \varphi(x)}$ domain. Also, by the argument of [PS, Section 3.3], the domain $D$ is regular with respect to the Dirichlet problem, and so is any $C^{b x \varphi(x)}$ domain.

We are now ready for our key step.

Lemma 2.8. There exists $b_{0} \in(0,1)$ with the property that for each $b \in$ $\left(0, b_{0}\right)$ there exists $a_{0}=a_{0}(b)$ such that if $a<a_{0}, u$ is nonnegative and harmonic in the domain $\Delta(0, \psi(a), \psi(a))$ and vanishes continuously on $\partial D \cap$ $\partial \Delta(0, \psi(a), \psi(a))$, and $h$ is as in $(2.5)$, then $u / h$ is uniformly continuous in $\Delta(0, a / 2, a / 2)$.

Proof. Let $r_{k}=2^{-k(\log k)^{2}}, x \in \partial \Delta(0, a / 2, a / 2)$,

$$
W_{k}=\Delta\left(x, r_{k}, K_{1} b r_{k} \varphi\left(r_{k}\right)\right), \quad V_{k}=\Delta\left(x, 2 r_{k}, 2 K_{1} b r_{k} \varphi\left(r_{k}\right)\right) .
$$

By the boundary Harnack principle, $\sup _{W_{k}}(u / h)<\infty$. Let

$$
O_{k}=\operatorname{Osc}_{W_{k}}(u / h)=\sup _{W_{k}}(u / h)-\inf _{W_{k}}(u / h) .
$$

Now for $k$ sufficiently large,

$$
W_{k} \subset \Delta(0, a, a), \quad r_{k} \geq 2 K_{1} r_{k+1} \log \left(r_{k+1}^{-1}\right)^{5}, \quad \text { and } \quad V_{k+1} \subset W_{k} .
$$

Observe $u / h$ is $P_{h}^{x}$-harmonic in $\Delta(0, \psi(a), \psi(a))$.

Let $U=A(u / h)+B$, where $A$ and $B$ are chosen so that $\sup _{W_{k}} U=1$ and $\inf _{W_{k}} U=0$. Then $U$ is $P_{h}^{x}$-harmonic in $W_{k}$. Since $V_{k+1} \subset W_{k}$, then $U$ is bounded by 0 and 1 in $V_{k+1}$. By looking at $1-U$ if necessary, we may suppose that $\sup _{\partial^{u} V_{k+1}} U \geq 1 / 2$. Note also that $h U$ is nonnegative and harmonic (with respect to $P^{x}$ ) in $W_{k}$.

Suppose $y \in W_{k+1}$. Then

$$
\begin{aligned}
U(y) & \geq E_{h}^{y}\left(U\left(X_{T\left(\partial V_{k+1}\right)}\right) ; X_{T\left(\partial V_{k+1}\right)} \in \partial^{u} V_{k+1}\right) \\
& \geq\left(\inf _{\partial^{u} V_{k+1}} U\right) P_{h}^{y}\left(X_{T\left(\partial V_{k+1}\right)} \in \partial^{u} V_{k+1}\right) .
\end{aligned}
$$

By Lemma 2.6

$$
P_{h}^{y}\left(X_{T\left(\partial V_{k+1}\right)} \in \partial^{u} V_{k+1}\right) \geq c_{1} \exp \left(-c_{2} b L_{2}\left(r_{k+1}\right)\right) .
$$

For some $x_{1} \in \partial^{u} V_{k+1}$ we have that $U\left(x_{1}\right) \geq 1 / 4$. Since both $h$ and $h U$ are nonnegative and harmonic in $W_{k}$, then by Lemma 2.5

$$
\begin{aligned}
h(z) & \leq c_{3} \exp \left(c_{4} b L_{2}\left(r_{k+1}\right)\right) h\left(x_{1}\right) \quad \text { and } \\
(h U)(z) & \geq c_{3}^{-1} \exp \left(-c_{4} b L_{2}\left(r_{k+1}\right)\right)(h U)\left(x_{1}\right)
\end{aligned}
$$

for $z \in \partial^{u} V_{k+1}$. Hence

$$
\begin{aligned}
U(z) & \geq c_{3}^{-2} \exp \left(-2 c_{4} b L_{2}\left(r_{k+1}\right)\right) U\left(x_{1}\right) \\
& \geq\left(c_{3}^{-2} / 4\right) \exp \left(-2 c_{4} b L_{2}\left(r_{k+1}\right)\right) .
\end{aligned}
$$


Recall that $U \leq 1$ on $W_{k+1}$. Substituting (2.8) and (2.9) in (2.7) gives

$$
\underset{W_{k+1}}{\operatorname{Osc} U} \leq \rho_{k+1} \underset{W_{k}}{\operatorname{Osc}} U
$$

where

$$
\rho_{k}=1-c_{5} \exp \left(-c_{6} b L_{2}\left(r_{k}\right)\right) .
$$

Recalling the definition of $U$, we then get $O_{k+1} \leq \rho_{k+1} O_{k}$.

Choose $b_{0}<1 \wedge c_{6}^{-1}$. If $b<b_{0}$, then $\sum_{k=k_{0}}^{\infty}\left(1-\rho_{k}\right)=\infty$ for any $k_{0}$, hence $\prod_{k=k_{0}}^{\infty} \rho_{k}=0$. The uniform continuity follows easily: if $b<b_{0}$, choose $a_{0}$ small as in Lemmas 2.2, 2.4-2.6. Then choose $k_{0}$ large as in (2.6). Given $\varepsilon$ choose $k_{1}>k_{0}$ so that $\prod_{k=k_{0}}^{k_{1}} \rho_{k}<\varepsilon$. For any two points $y_{1}, y_{2}$ in $\Delta\left(x, r_{k_{1}+1}, K_{1} r_{k_{1}+1} \varphi\left(r_{k_{1}+1}\right)\right)$,

$$
\begin{aligned}
& \left|(u / h)\left(y_{1}\right)-(u / h)\left(y_{2}\right)\right| \leq \underset{W_{k_{1}+1}}{\operatorname{Osc}}(u / h) \leq\left(\prod_{k=k_{0}+1}^{k_{1}+1} \rho_{k}\right) \underset{W_{k_{0}}}{\operatorname{Osc}}(u / h) \\
& \leq \varepsilon \sup _{\partial \Delta(0, a, a)}(u / h) \text {. }
\end{aligned}
$$

We now turn to the Martin boundary. Suppose $D \subset \mathbb{R}^{d}$, fix a base point $x_{0} \in D$ and define the Martin kernel

$$
K(y, x)=G_{D}(y, x) / G_{D}\left(y, x_{0}\right)
$$

for $x, y \in D, y \neq x_{0}$, where $G_{D}$ is the Green function for the domain $D$. There is a unique (up to a homeomorphism) compactification $D^{M}$ of $D$ with the property that $K$ has a continuous extension to $\left(D^{M}-\left\{x_{0}\right\}\right) \times D$ and $K\left(z_{1}, \cdot\right) \equiv K\left(z_{2}, \cdot\right)$ if and only if $z_{1}=z_{2} \in D^{M}$ [Do, 1 XII 3]. The set $\partial^{M} D \stackrel{\text { df }}{=} D^{M}-D$ is called the Martin boundary of $D$.

A positive harmonic function $h$ is minimal if whenever $g$ is a positive harmonic function in $D$ with $g \leq h$, then $g \equiv c h$ for some constant $c$. The set of all points $z \in \partial^{M} D$ such that $K(z, \cdot)$ is a minimal harmonic function is called the minimal Martin boundary and denoted $\partial_{1}^{M} D$.

Theorem 1.1 can then be expressed as follows. If $D$ is a bounded $C^{\gamma}$ domain with $\gamma$ given by (1.1), $b$ sufficiently small, then the function $K$ has a continuous extension to $\left(\bar{D}-\left\{x_{0}\right\}\right) \times D$, we have $K\left(z_{1}, \cdot\right) \equiv K\left(z_{2}, \cdot\right)$ if and only if $z_{1}=z_{2}$ and, moreover, $\partial_{1}^{M} D=\partial^{M} D$.

Suppose from now on that $D$ is a $C^{b x \varphi(x)}$ domain, $b$ less than the $b_{0}$ of Lemma 2.8.

Proof of Theorem 1.1. Pick $x_{0}, x \in D$. For $z \in \partial D$ pick a neighborhood $U$ and a coordinate system $C S$ so that

$$
U \cap D=\left\{y: y^{d}>\Gamma(\tilde{y}) \text { if } y=\left(\tilde{y}, y^{d}\right) \text { in } C S\right\} \cap U .
$$

Without loss of generality we may suppose that our coordinate system was chosen so that $z=0$. Take $a_{0}$ small enough so that $\Delta(0, \psi(a), \psi(a)) \subset U \cap D$ and that $x_{0}, x \notin \partial \Delta(0, \psi(a), \psi(a))$ if $a<a_{0}$. Define $h$ as in (2.5) and suppose $u$ and $v$ are positive and harmonic in $\Delta(0, \psi(a), \psi(a))$, vanishing continuously on $\partial D \cap \partial \Delta(0, \psi(a), \psi(a))$. By Remark $2.7 h$ also vanishes 
continuously on $\partial D \cap \partial \Delta(0, \psi(a), \psi(a))$. By Lemma $2.8 u / h$ and $v / h$ are uniformly continuous in $\Delta(0, a / 2, a / 2)$.

If $x_{1} \in \Delta(0, a, a)$, then $u\left(x_{1}\right) / h\left(x_{1}\right) \in(0, \infty)$, clearly, and by the boundary Harnack principle, there exists a constant $c_{1}$ such that

$$
c_{1}^{-1}(u / h)\left(x_{1}\right) \leq(u / h)(y) \leq c_{1}(u / h)\left(x_{1}\right), \quad y \in \Delta(0, a, a) .
$$

The same holds for $v / h$. Therefore we see that $u / v=(u / h) /(v / h)$ is uniformly continuous in $\Delta(0, a / 2, a / 2)$.

We now choose $u(y)=G_{D}(x, y)$ and $v(y)=G_{D}\left(x_{0}, y\right)$. Since $D$ is regular, $u, v$ vanish continuously on $\partial D$. So for a fixed $x$, the function $K(y, x)=$ $u(y) / v(y)$ has a continuous extension to $\bar{D}-\left\{x_{0}\right\}$. For each $z \in \bar{D}-\left\{x_{0}\right\}$, the function $K(z, \cdot)$ is positive and harmonic in $D-\{z\}$. This, the Harnack inequality, and the fact that $K\left(z, x_{0}\right)=1$ for every $z$ may be used to show that the family of functions $\{K(z, \cdot), z \notin B(x, r)\}$ is uniformly continuous on $B(x, r / 2)$ if $B(x, r) \subset D$. This implies easily that $K$ is jointly continuous on $\left(\bar{D}-\left\{x_{0}\right\}\right) \times D$, except on the diagonal.

We next need to show that the Martin kernels corresponding to two distinct points $w, z \in \partial D$ are distinct. The proof of this follows exactly the proof of Theorem 4.4 of [BB1], using the boundary Harnack principle of [BB2] or [BBB] in place of Theorem 1.1 of [BB1].

Finally, we need to show that each $K(z, \cdot)$ is a minimal harmonic function. To see this, note that the proof of Theorem 4.5 of [BB1] may be followed virtually word for word. This completes the proof of Theorem 1.1.

Remark 2.9. The proof of Theorem 4.4 of [BB1] depends only on the boundary Harnack principle and not Lemma 2.8. So a Martin boundary point cannot correspond to more than one Euclidean boundary point in any domain for which the boundary Harnack principle holds, e.g., Hölder domains. On the other hand, the first example of $\S 5$ of [BB2] is that of a domain above the graph of a continuous function where the boundary Harnack principle does not hold; one can show that this domain has a Martin boundary point which corresponds to more than one Euclidean boundary point.

The proof of Theorem 1.1 shows, in particular, the following.

Theorem 2.10. Suppose $D$ is as in Theorem 1.1. If A compact is contained in $V$ open and $u$ and $v$ are positive and harmonic in $D$ and vanish continuously on $\partial D \cap V$, then $u / v$ is uniformly continuous in $A \cap D$.

Remark 2.11. Define

$$
\omega(x, A)=P^{x}\left(X_{T(\partial \Delta)} \in A\right), \quad x \in D, A \subset \partial D,
$$

the harmonic measure of the set $A$ with respect to the domain $D$. By Theorem 1 XII 10 of [Do],

$$
\omega(x, A)=\int_{A} K(y, x) \omega\left(x_{0}, d y\right) \text { for all } A \subset \partial D .
$$

Consequently $\omega(x, A) / \omega\left(x_{0}, A\right) \rightarrow K(z, x)$ whenever $A$ decreases to $\{z\}$. This is an extension to $C^{b x \varphi(x)}$ domains of one of the main results of [HW].

\section{DiVERGENCE FORM OPERATORS}

In this section we consider the Martin boundary with respect to $L$-harmonic functions, where $L$ is a uniformly elliptic operator in divergence form. We let 
$L$ be the operator defined by

$$
L f(x)=\sum_{i, j=1}^{d} \frac{\partial}{\partial x^{i}}\left(a_{i j}(x) \frac{\partial f}{\partial x^{j}}\right)(x),
$$

where we assume the $a_{i j}$ are bounded and uniformly elliptic:

(3.1) there exists $c_{L}$ such that

$$
c_{L}^{-1} \sum_{i=1}^{d}\left(y^{i}\right)^{2} \leq \sum_{i, j=1}^{d} a_{i j}(x) y^{i} y^{j} \leq c_{L} \sum_{i=1}^{d}\left(y^{i}\right)^{2}, \quad\left(y^{1}, \ldots, y^{d}\right) \in \mathbb{R}^{d} .
$$

We also assume the $a_{i j}$ are smooth, but the estimates we obtain will depend on the $a_{i j}$ 's only through the bound $c_{L}$ and not on the smoothness of the $a_{i j}$ 's.

Let $\left(X_{t}, P^{x}\right)$ be the strong Markov process corresponding to $L$. Let

$$
F_{a}=\{x \in D: \delta(x)<a\}
$$

All potential-theoretic objects, e.g., the Green function, are defined relative to $L$ in this section. See [BB2, §4], for a summary of estimates related to divergence form operators.

Lemma 3.1. The analog of Lemma 2.1 holds.

Proof. By Lemma 2.1 of [BB2] there exist $c_{1}, c_{2}$ such that

$$
\left|B(x, 2 a) \cap F_{a}^{c}\right| /|B(x, 2 a)| \geq c_{1} \text { if } a<c_{2},
$$

where $|\cdot|$ denotes Lebesgue measure. By the remarks and results of $\S 4$ of [BB2], $\left.P^{x}\left(T\left(F_{a}^{c}\right)\right)<T(\partial B(x, 2 a))\right) \geq c_{3}$, with $c_{3}$ independent of $x$ and $a$. Using this estimate we then follow the proof of Lemma 2.2 of [BB2].

Using Moser's Harnack principle (see [BB2, §4]), instead of the usual Harnack's inequality, Lemmas $2.3-2.5$ go through as before. The main change required is in the proof of Lemma 2.6.

If $G_{D}(\cdot, \cdot)$ denotes the Green function for the domain $D$ with respect to $L$, let $g(x)=G_{\mathbf{R}^{d}}(0, x)$, the Green function in $\mathbb{R}^{d}$ with pole at 0 .

Lemma 3.2. There exists $\beta$ depending only on the constant $c_{L}$ such that

$$
P_{g}^{x}(T(\{0\})<T(\partial B(0,1))) \geq 1 / 2
$$

whenever $x \in B(0, \beta)$.

Proof. By [FS] there exists $c_{1}$ depending only on $c_{L}$ such that

$$
c_{1}|x|^{2-d} \leq g(x) \leq c_{1}^{-1}|x|^{2-d} .
$$

For $a>0$ let $A(a)=\{y: g(y)=a\}$. By (3.3), if $a_{1}$ is sufficiently large, $A\left(a_{1}\right) \subset B(0,1)$. Again using (3.3), we may take $\beta$ small (depending only on $c_{L}$ and $a_{1}$ so that $B(0, \beta) \subset\left\{y: g(y) \geq 2 a_{1}\right\}$.

Suppose $x \in B(0, \beta)$. If $a_{2}>2 a_{1} \vee 2 g(x)$ is large enough so that $A\left(a_{2}\right) \subset$ $B(0, \beta)$, then

$$
\begin{aligned}
P_{g}^{x}\left(T\left(A\left(a_{2}\right)\right)<T\left(A\left(a_{1}\right)\right)\right) & \geq \frac{E^{x}\left(g\left(X_{T\left(A\left(a_{2}\right)\right)}\right) ; T\left(A\left(a_{2}\right)\right)<T\left(A\left(a_{1}\right)\right)\right)}{g(x)} \\
& \geq \frac{a_{2}}{g(x)} P^{x}\left(g\left(X_{t}\right) \text { hits } a_{2} \text { before hitting } a_{1}\right) \\
& =\frac{a_{2}}{g(x)} \frac{g(x)-a_{1}}{a_{2}-a_{1}} \geq 1-a_{1} / g(x) \geq 1 / 2,
\end{aligned}
$$


using the fact that $g\left(X_{t}\right)$ is a martingale up until time $T\left(A\left(a_{2}\right)\right)$. Now let $a_{2} \rightarrow \infty$.

Suppose now that $D$ is the region above a $C^{b x \varphi(x)}$ function, $a<a_{0}, H_{2}$ and $h$ defined as in (2.2) and (2.5).

Lemma 3.3. If $x \in \partial \Delta(0, a, a)$ and $K_{1} b a \varphi(a) \leq R \leq 2 K_{1} b a \varphi(a)$, then

$$
P_{h}^{x}\left(H_{2}\right) \geq c_{1} \exp \left(-c_{2} b L_{2}(a)\right) .
$$

Proof. Suppose $v \in D$ with $\operatorname{dist}(v, \Delta(0,2 a, 2 R)) \in(6 R, 12 R)$. Write $\Delta_{1}$ for $\Delta(0,2 a, 2 R)$,

$\Delta_{2}=\Delta(v, \delta(v)+a, 2 R)-\Delta(v, \delta(v)-a, 2 R), \quad \partial^{u} \Delta_{2}=\partial^{u} \Delta(v, \delta(v)+a, 2 R)$.

If $G(x, y)=G_{\mathbb{R}^{d}}(x, y)$, note that there exists $c_{3}$ depending only on $c_{L}$ such that

$$
c_{3}^{-1} \leq G(z, w) / G(z, v) \leq c_{3} \quad \text { and } \quad c_{3}^{-1} \leq G(v, z) / G(v, x) \leq c_{3}
$$

for $z \in \partial \Delta(0,2 a, 2 R), w \in \partial \Delta(v, 2 a, 2 R)$ and $x \in \partial \Delta(0, a, R)$.

Now suppose $z \in \partial^{u} \Delta_{1}, w \in \partial^{u} \Delta_{2}$. Let $c_{4}=\beta d(z) / 2$, where $\beta$ is as in Lemma 3.2. Arguing as in Lemmas 2.2, 2.3, and 2.5, $P^{w}\left(T\left(B\left(z, c_{4}\right)\right)<T(\partial D)\right)$ $\geq c_{5} \exp \left(-c_{6} b L_{2}(a)\right)$. By (3.3) $G(z, x) / G(z, w) \geq c_{7}$ for $x \in \partial B\left(z, c_{4}\right)$. Hence $P_{G(z, .)}^{w}\left(T\left(B\left(z, c_{4}\right)\right)<T(\partial D)\right) \geq c_{8} \exp \left(-c_{6} b L_{2}(a)\right)$.

If $x \in \partial B\left(z, c_{4}\right)$, then $P_{G(z, \cdot)}^{x}(T(\{z\})<T(\partial D))>c_{9}$; this follows from Lemma 3.2 and a scaling argument. So by the strong Markov property for the process $\left(X_{t}, P_{G(z, \cdot)}^{w}\right)$, we see that

$$
P_{G(z, \cdot)}^{w}(T(\{z\})<T(\partial D))>c_{10} \exp \left(-c_{6} b L_{2}(a)\right) .
$$

We next turn to the proof of Theorem 3.2 of [BB2], using $\partial^{u} \Delta$ instead of $\partial^{g} \Delta$. Rather than repeating the rather long proof, we sketch the changes necessary. By (3.3), $c_{3}, c_{4}, c_{6}$, and $c_{9}$ of that proof may be taken to be independent of $a$. By (3.4) $c_{5}$ of that proof is greater than or equal to $c_{10} \exp \left(-c_{6} b L_{2}(a)\right)$. So from the conclusion of that theorem, we get

$$
P_{G_{D}(v, \cdot)}^{x}\left(H_{2}\right) \geq c_{11} \exp \left(-c_{6} b L_{2}(a)\right)
$$

or

$$
E^{x}\left(G_{D}\left(X_{T\left(\partial \Delta_{1}\right)}, v\right) ; H_{2}\right) \geq c_{11} \exp \left(-c_{6} L_{2}(a)\right) G_{D}(x, v) .
$$

Now let $D_{n}=\{v \in D: \operatorname{dist}(v, \partial \Delta(0,2 a, 2 R))<9 R\}-J_{n}$, where $J_{n}=\{x$ : $d(x)<1 / n\}$. Note $\bar{D}_{n} \subset D$. By standard results on balayage and réduites, if $h_{n}$ is $L$-harmonic in $D_{n}$, then $h_{n}(x)=\int G_{D}(x, v) \mu_{n}(d v)$ for some measure $\mu_{n}$ on $\partial D_{n}$. See [Do, p. 160], for the proofs in the case of the Laplacian; since the Green function corresponding to the operator $L$ is symmetric, the proofs go through in the present case. If in addition $h_{n}$ has boundary values 0 on $\partial J_{n}$, we have that $\mu_{n}$ is supported on $\partial D_{n}-\partial J_{n}$. Integrating (3.5) with respect to $\mu_{n}$, we get

$$
E^{x}\left(h_{n}\left(X_{T\left(\partial \Delta_{1}\right)}\right) ; H_{2}\right) \geq c_{11} \exp \left(-c_{6} b L_{2}(a)\right) h_{n}(x)
$$

for such $h_{n}$ provided $n$ is large enough so that $x \in D_{n}$.

Finally let $h_{n}(x)=E^{x}\left(h\left(X_{T\left(\partial D_{n}\right)}\right) ; X_{T\left(\partial D_{n}\right)} \notin \partial J_{n}\right)$. Then $h_{n}$ is $L$-harmonic on $D_{n}$ and 0 on $\partial J_{n}$. Letting $n \rightarrow \infty$ and using the fact that $h$ is bounded 
by 1 , we get that $h_{n}$ converges to $h$ in $\Delta(0,3 a, 3 R)$ by dominated convergence. Then letting $n \rightarrow \infty$ in (3.6) and using dominated convergence again, we obtain our result.

Theorem 3.4. Theorem 1.1 holds for the Martin boundary with respect to the operator $L$.

Proof. We replace the use of Lemma 2.6 by Lemma 3.3, we use Moser's Harnack inequality instead of the usual one, and we use the estimates of $\S 4$ of [BB2] in the proof of Theorem 4.1 of [BB1]. With these changes the proofs in $\S 2$ go through.

\section{Counterexample}

For each $b_{1}>0$ we will construct a bounded $C^{\gamma}$ domain $D$ with

$$
\gamma(x)=b_{1} x \log \log (1 / x)
$$

and such that the Euclidean and Martin compactifications of $D$ are different. Our example is 3 -dimensional. It is easy to see that $D \times(0,1)^{d-3}$ provides a $d$-dimensional example of the same kind, for $d \geq 4$. The construction will take up the rest of the section and will be divided into steps.

In this section, $P^{x}, P_{h}^{x}$, etc. will refer to the standard and conditioned Brownian motion.

Step 1. First we will define $D$ and argue that it is a $C^{\gamma}$ domain. Recall that $L_{2}(x)$ denotes $\log \log (1 / x)$. Let

$$
f\left(x^{1}, x^{3}\right)=a\left|x^{1}\right| / L_{2}\left(\left|x^{1}\right|\right)+b x^{3} / L_{2}\left(x^{3}\right)
$$

for $x^{1} \in \mathbb{R}, x^{3}>0$. We will use the convention $1 / L_{2}(0)=0$. The values of $a$ and $b$ will be specified later. At this point it will suffice to say that $a$ and $b$ should be thought of as large numbers satisfying $0<b<a<\infty$. Let

$$
\begin{gathered}
D=\left\{x \in \mathbb{R}^{3}:\left|x^{2}\right|<f\left(x^{1}, x^{3}\right), 0<x^{3} \leq 1 / 100-\left|x^{1}\right| / 10\right\} \\
\cup\left\{x \in \mathbb{R}^{3}:\left|x^{2}\right|<1,1 / 100-\left|x^{1}\right| / 10<x^{3}<1-\left|x^{1}\right| / 10, x^{3}>0\right\}, \\
\partial^{u} D=\left\{x \in \partial D: x^{3}=1-\left|x^{1}\right| / 10\right\} .
\end{gathered}
$$

It is evident that for every closed ball $B(0, r)$ with $r$ positive and small, $D-B(0, r)$ is a Lipschitz domain. We have to show that it is a $C^{\gamma}$ domain in a neighborhood of 0 . Our estimates in this and subsequent steps hold only locally near 0 but this does not cause any loss of generality.

Let $g$ be the inverse function of $t \rightarrow t / L_{2}(t)$, i.e., $t=g(s)$ if $s=t / L_{2}(t)$, for all $t \in[0,1 / e)$. It is elementary to check that

$$
g\left(c_{1} t\right) \leq 2 c_{1} t L_{2}(t)
$$

for small $t$.

The equation $x^{2}=f\left(x^{1}, x^{3}\right)$ describes the surface $\left\{x \in \partial D: x^{1}>0, x^{2}>\right.$ $\left.0, x^{3}>0\right\}$ near 0 . We may write the equation in an equivalent way as

$$
b x^{3} / L_{2}\left(x^{3}\right)=x^{2}-a x^{1} / L_{2}\left(x^{1}\right)
$$

or

$$
x^{3}=g\left((1 / b)\left(x^{2}-a x^{1} / L_{2}\left(x^{1}\right)\right)\right) .
$$


Hence, for small $r>0, \partial D \cap B(0, r)$ is given by

$$
x^{3}=g\left((1 / b)\left(\left|x^{2}\right|-a\left|x^{1}\right| / L_{2}\left(\left|x^{1}\right|\right)\right)^{+}\right) \stackrel{\mathrm{df}}{=} g_{1}\left(x^{1}, x^{2}\right) .
$$

The function $\left(x^{1}, x^{2}\right) \rightarrow\left(\left|x^{2}\right|-a\left|x^{1}\right| / L_{2}\left(\left|x^{1}\right|\right)\right)^{+}$is Lipschitz, so (4.1) implies that

$$
\begin{aligned}
\left|x^{3}-z^{3}\right| & =\left|g_{1}\left(x^{1}, x^{2}\right)-g_{1}\left(z^{1}, z^{2}\right)\right| \\
& \leq\left(c_{2} / b\right)\left|\left(x^{1}, x^{2}\right)-\left(z^{1}, z^{2}\right)\right| \cdot L_{2}\left(\left|\left(x^{1}, x^{2}\right)-\left(z^{1}, z^{2}\right)\right|\right)
\end{aligned}
$$

for small $|x|$ and $|z|$. We conclude that $D$ is a $C^{\gamma}$ domain with $\gamma(x)=$ $b_{1} x L_{2}(x)$, provided we take $b>c_{2} / b_{1}$.

Step 2. This step is devoted to some preliminary estimates of the Green function in $D$ which are very crude.

Fix a base point $x_{0}=(0,0,3 / 4)$ and let $h(x)=G_{D}\left(x, x_{0}\right)$, the Green function with pole at $x_{0}$. We will compare $h$ to a harmonic function $h_{1}$ which is positive in $D$ with boundary values 1 on $\partial^{u} D$ and 0 elsewhere.

First we will show that $h_{1}\left(x^{1}, x^{2}, x^{3}\right)$ is increasing in $x^{1}$ for $x^{1}>0$. Let $D_{1} \stackrel{\text { df }}{=}\left\{x \in D: x^{1}>0\right\}$. Observe that $\partial h_{1} / \partial x^{1} \geq 0$ on $\partial^{u} D \cap \partial D_{1}$ because $h_{1} \leq 1$ in $D$ and $h_{1}=1$ on $\partial^{u} D$. For similar reasons, $\partial h_{1} / \partial x^{1} \geq 0$ on the remaining part of $\partial_{1} D \cap \partial D$. For $x \in \partial D_{1}$ with $x^{1}=0$ we have $\partial h_{1} / \partial x^{1}=0$, by symmetry. Since $\partial h_{1} / \partial x^{1}$ is harmonic in $D$, we obtain $\partial h_{1} / \partial x^{1} \geq 0$ in $D$ and, therefore, $h_{1}\left(x^{1}, x^{2}, x^{3}\right)$ is increasing in $x^{1}$ for $x^{1}>0$.

A similar argument shows that $h_{1}\left(x^{1}, x^{2}, x^{3}\right)$ is decreasing in $x^{2}$ for $x^{2}>$ 0 ; one has to consider the boundary values of $\partial h_{1} / \partial x^{2}$ in $\left\{x \in D: x^{2}>0\right\}$. Finally, note that $h_{1}\left(x^{1}, x^{2}, x^{3}\right)$ is increasing in $x^{3}$. In order to see this, examine the boundary values of $\partial h_{1} / \partial x^{3}$ in $D$.

For an integer $k>0$, let

$$
\begin{aligned}
F_{k}= & \left\{x \in D:\left|x^{1}\right|=2^{-k}, x^{3} \leq 2^{-k}\right\} \\
& \cup\left\{x \in D:\left|x^{1}\right| \leq 2^{-k}, x^{3}=2^{-k}\right\}, \\
V= & \left\{x \in D:\left|x^{1}\right| \in(3 / 8,5 / 8), x^{3}<5 / 8\right\} \\
& \cup\left\{x \in D:\left|x^{1}\right|<5 / 8, x^{3} \in(3 / 8,5 / 8)\right\} .
\end{aligned}
$$

The functions $h$ and $h_{1}$ are positive and harmonic in $V$ and vanish on $\partial D \cap$ $\partial V$. Fix some $y_{0} \in F_{1}$. The boundary Harnack principle (see Remark 2.7) implies that

$$
\frac{h(x)}{h_{1}(x)} \geq c_{3} \frac{h\left(y_{0}\right)}{h_{1}\left(y_{0}\right)}
$$

and

$$
\frac{h_{1}(x)}{h(x)} \geq c_{3} \frac{h_{1}\left(y_{0}\right)}{h\left(y_{0}\right)}
$$

for all $x \in F_{1}$. Let

$$
c_{4}=c_{3} \min \left(h\left(y_{0}\right) / h_{1}\left(y_{0}\right), h_{1}\left(y_{0}\right) / h\left(y_{0}\right)\right) .
$$

For $|x|<1 / 2, x \in D$,

$$
h(x)=E^{x}\left(h\left(X\left(T\left(F_{1}\right)\right)\right)\right) \geq c_{4} E^{x}\left(h_{1}\left(X\left(T\left(F_{1}\right)\right)\right)\right)=c_{4} h_{1}(x) .
$$


For similar reasons, $h_{1}(x) \geq c_{4} h(x)$. These two inequalities and the monotonicity properties of $h_{1}$ mentioned above imply that

$$
h\left(y^{1}, y^{2}, y^{3}\right) \leq c_{5} h\left(x^{1}, 0, x^{3}\right),
$$

for $c_{5}=c_{4}^{-2}<\infty$ and all $x, y \in D,|x|<1 / 2,|y|<1 / 2,\left|y^{1}\right| \leq x^{1}, y^{3} \leq x^{3}$.

Step 3. We proceed to obtain much more accurate estimates of the function $h$.

We start with a lower bound for $h(x)$ for $x \in D$ with $2^{-k}>x^{1}>2^{-k} / 40$, $x^{2}=0,2^{-k}>x^{3}>2^{-k} / 40$. Let $z_{k}=\left(2^{-k}, 0,2^{-k}\right)$. The points $x$ and $z_{k}$ may be connected by a chain of balls $B_{1}, B_{2}, \ldots, B_{n+m}$ with the following properties. Each ball $B_{j}$ has radius $r=a\left(2^{-k} / 40\right) / 2 L_{2}\left(2^{-k} / 40\right)$. The centers $y_{j}$ of $B_{j}$ are chosen so that $y_{j}^{2}=0$ for all $j$. We let $y_{1}=x, y_{j}^{3}=y_{1}^{3}$ for $j=1, \ldots, n, y_{j}^{1}=y_{j-1}^{1}+r / 2$ for $j=2, \ldots, n-1, y_{n}^{1} \leq y_{n-1}^{1}+r / 2$, $y_{n}^{1}=2^{-k}$. Note that

$$
n \leq 2^{-k} /(r / 2)+1 \text {. }
$$

Let $y_{j}^{1}=y_{n}^{1}=2^{-k}$ for $j \geq n+1, y_{j}^{3}=y_{j-1}^{3}+r / 2$ for $j=n+1, \ldots, n+m-1$, $y_{n+m}^{3} \leq y_{n+m-1}^{3}+r / 2, y_{n+m}=z_{k}$.

We have $m \leq 2^{-k} /(r / 2)$ and the total number $n+m$ of balls in the chain does not exceed

$$
2 \cdot 2^{-k} /(r / 2)+1 \leq(8 \cdot 40 / a) L_{2}\left(2^{-k} / 40\right)+1 \leq c_{6} \log k
$$

for large $k$. We may take $c_{6}=16 \cdot 40 / a$.

All the balls $B_{j}$ are contained in $D-\left\{x_{0}\right\}$ so we may apply the Harnack principle in each of them. We obtain

$$
\begin{aligned}
h(x) & \geq h\left(z_{k}\right) c_{7}^{n+m} \geq h\left(z_{k}\right) \exp \left(-c_{8}(n+m)\right) \\
& \geq h\left(z_{k}\right) \exp \left(-c_{8} c_{6} \log k\right)=h\left(z_{k}\right) k^{-c_{8} c_{6}}=h\left(z_{k}\right) k^{-c_{9} / a} .
\end{aligned}
$$

Next we will find an upper bound on $h(x)$ for $x \in D$ with $x^{1}=0$, $x^{3} \in\left[2^{-k} / 4,2^{-k} / 2\right]$. Recall that $z_{k}=\left(2^{-k}, 0,2^{-k}\right)$. Suppose that $b / a \in$ $\left(2^{-j-1}, 2^{-j}\right]$, where $j>0$. If $y \in D$ with

$$
\left|\left(y^{1}, y^{3}\right)-\left(x^{1}, x^{3}\right)\right| \in\left[2^{-k-n}, 2^{-k-n+1}\right), \quad 2 \leq n \leq j,
$$

then let

$$
\begin{array}{r}
A(y)=\left\{z \in \mathbb{R}^{3}:\left|z^{1}-y^{1}\right|<a 2^{-k-n} / L_{2}\left(2^{-k-n}\right),\left|z^{2}\right|<8 a 2^{-k-n} / L_{2}\left(2^{-k-n}\right),\right. \\
\left.\left|z^{3}-y^{3}\right|<a 2^{-k-n} / L_{2}\left(2^{-k-n}\right)\right\},
\end{array}
$$

and let $K(y)$ be the ball with radius $a 2^{-k-n} / 2 L_{2}\left(2^{-k-n}\right)$ and center

$$
\left(y^{1}, y^{2}+7 a 2^{-k-n} / L_{2}\left(2^{-k-n}\right), y^{3}\right) \text {. }
$$

We will show that $K(y) \subset A(y)-D$. Note that for $k$ large

$$
\begin{gathered}
y^{3} \leq 2^{-k} / 2+2^{-k-n+1} \leq 2^{-k} / 2+2^{-k-2+1}=2^{-k}, \\
2^{-j} y^{3} \leq 2^{-k-j}<2^{-k-n+1}, \\
L_{2}\left(y^{3}\right) \geq L_{2}\left(2^{-k}\right) \geq L_{2}\left(2^{-k-n+1}\right) / 2 .
\end{gathered}
$$

These inequalities and the fact that $b / a \leq 2^{-j}$ imply for $k$ large and $y, n$ as above,

$$
b y^{3} / L_{2}\left(y^{3}\right) \leq a 2^{-j} y^{3} / L_{2}\left(y^{3}\right) \leq a 2^{-k-n+1} / L_{2}\left(y^{3}\right) \leq 2 a 2^{-k-n+1} / L_{2}\left(2^{-k-n+1}\right) .
$$


It follows that

$$
\begin{aligned}
f\left(y^{1}, y^{3}\right) & \leq a\left|y^{1}\right| / L_{2}\left(\left|y^{1}\right|\right)+b y^{3} / L_{2}\left(y^{3}\right) \\
& \leq a 2^{-k-n+1} / L_{2}\left(2^{-k-n+1}\right)+2 a 2^{-k-n+1} / L_{2}\left(2^{-k-n+1}\right) \\
& =3 a 2^{-k-n} / L_{2}\left(2^{-k-n}\right) .
\end{aligned}
$$

Now it is easy to check that $K(y) \subset A(y)-D$. This and Brownian scaling imply that

$$
P^{y}\left[T\left(D^{c}\right)>T\left(A^{c}(y)\right)\right]<P^{y}\left(\left[(K(y))>T\left(A^{c}(y)\right)\right]=c_{10}<1 .\right.
$$

Let

$$
\begin{aligned}
& T_{0}=\inf \left\{t>0:\left|x-X_{t}\right| \geq 2^{-k-j}\right\}, \\
& T_{n}=\inf \left\{t>T_{n-1}: X_{t} \notin A\left(X\left(T_{n-1}\right)\right)\right\}, \quad n \geq 1 .
\end{aligned}
$$

A repeated application of the strong Markov property at the stopping times $T_{n}$ gives

$$
P^{x}\left(T\left(D^{c}\right)>T_{n}\right) \leq c_{10}^{n} .
$$

Recall from Step 2 that $h(z) \leq c_{5} h\left(z_{k}\right)$ for all $z \in F_{k}$ (see (4.2)). Note that at least $2^{-k-n} /\left(a 2^{-k-n} / L_{2}\left(2^{-k-n}\right)\right)-1$ stopping times $T_{m}$ must occur between the hitting times of

$$
\left\{y \in D:\left|\left(y^{1}, y^{3}\right)-\left(x^{1}, x^{3}\right)\right|=2^{-k-n}\right\}
$$

and

$$
\left\{y \in D:\left|\left(y^{1}, y^{3}\right)-\left(x^{1}, x^{3}\right)\right|=2^{-k-n+1}\right\},
$$

assuming the process starts from $x$ and does not hit $D^{c}$. Hence, $P^{x}$-a.s.,

$$
\left\{T\left(D^{c}\right)>T\left(F_{k}\right)\right\} \subset\left\{T\left(D^{c}\right)>T_{m}\right\},
$$

where

$$
\begin{aligned}
m & \geq \sum_{n=2}^{j}\left[2^{-k-n} /\left(a 2^{-k-n} / L_{2}\left(2^{-k-n}\right)\right)-1\right] \\
& \geq(j-1)[(1 / 2 a) \log k-1] \\
& \geq[\log (a / b) / \log 2-2](1 / 4 a) \log k
\end{aligned}
$$

for large $k$. We obtain

$$
\begin{aligned}
h(x) & =E^{x}\left(h\left(X\left(T_{F_{k}}\right)\right) 1_{\left\{T\left(D^{c}\right)>T\left(F_{k}\right)\right\}}\right) \\
& \leq c_{5} h\left(z_{k}\right) P^{x}\left(T\left(D^{c}\right)>T\left(F_{k}\right)\right) \\
& \leq c_{5} h\left(z_{k}\right) P^{x}\left(T\left(D^{c}\right)>T_{m}\right) \\
& \leq c_{5} h\left(z_{k}\right) c_{10}^{m} \\
& \leq c_{5} h\left(z_{k}\right) k^{-c_{11}[\log (a / b) / \log 2-2] / 4 a} .
\end{aligned}
$$

Now choose $a$ so that $0<b<a$ and

$$
c_{11}[\log (a / b) / \log 2-2] / 4 a \geq c_{9} / a+2
$$

Then

$$
h(x) \leq k^{-c_{9} / a-2} c_{5} h\left(z_{k}\right)
$$

for large $k$ and $x$ such that $x^{1}=0, x^{3} \in\left[2^{-k} / 4,2^{-k} / 2\right]$. 
Step 4. Let

$$
H=\left\{x \in D: x^{3}>10\left|x^{1}\right|\right\}, \quad J=\left\{x \in \mathbb{R}^{3}: x^{3}=10 x^{1}\right\} .
$$

If $x \in \mathbb{R}^{3}$, let $\mathscr{S}(x)$ denote the point obtained by reflecting across the plane $J$. We will show that $\widetilde{H} \stackrel{\text { df }}{=} \mathscr{S}(H) \subset D$ for some choice of $a$ and $b$.

Suppose that $\lambda>0$ and let $N=\left\{x \in H: x^{3}=\lambda\right\}$. Note that $x^{2} \leq$ $f(\lambda / 10, \lambda)$ for $x \in N$. In order to show that $\widetilde{H} \subset D$, it will suffice to prove that $\tilde{N} \stackrel{\text { df }}{=} \mathscr{S}(N) \subset D$ for every $\lambda>0$. The set $\widetilde{N}$ lies in a plane whose projection on the $\left(x^{1}, x^{3}\right)$-plane is a straight line $L$ with the slope $\tan (2 \arctan (1 / 10))$. We will show that $f\left(x^{1}, x^{3}\right)$ increases when $\left(x^{1}, x^{3}\right) \in L, x^{1}>x^{3} / 10$, and $x^{1}$ increases. For $x^{1}>0, x^{3}>0$,

$$
\begin{aligned}
& \frac{\partial}{\partial x^{1}} f\left(x^{1}, x^{3}\right)=a\left(\frac{1}{\log \left(1 / x^{1}\right)\left(L_{2}\left(x^{1}\right)\right)^{2}}+\frac{1}{L_{2}\left(x^{1}\right)}\right) \\
& \frac{\partial}{\partial x^{3}} f\left(x^{1}, x^{3}\right)=b\left(\frac{1}{\log \left(1 / x^{3}\right)\left(L_{2}\left(x^{3}\right)\right)^{2}}+\frac{1}{L_{2}\left(x^{3}\right)}\right)
\end{aligned}
$$

For small $x^{1}, x^{3}$ such that $x^{1}>x^{3} / 10$ we have

$$
\frac{\partial}{\partial x^{1}} f / \frac{\partial}{\partial x^{3}} f>(a / 2 b) \log \left(1 / x^{3}\right) / \log \left(10 / x^{3}\right)>a / 4 b \text {. }
$$

Thus, if we assume that $a / b$ is large then $f$ is increasing along $L$ for $x^{1}>$ $x^{3} / 10$ which completes the proof of the fact that $\widetilde{H} \subset D$.

Note that we may assume that $a / b$ is large without contradicting the assumptions on $a$ and $b$ imposed in Steps 1 and 3.

Step 5. Let

$$
\begin{aligned}
M_{k} & =\left\{x \in D: x^{1}=0, x^{3} \in\left[2^{-k} / 4,2^{-k} / 2\right]\right\}, \\
\widetilde{J} & =\left\{x \in \mathbb{R}^{3}: x^{3}=-10 x^{1}\right\}, \quad K=J \cup \widetilde{J} .
\end{aligned}
$$

Let $Q_{k}$ be the union of the images of $M_{k}$ by the reflections with respect to the planes $J$ and $\widetilde{J}$.

Consider an exit system of Brownian motion $X$ from $K$ (see [Bu] or [S] for the definition and properties of exit systems). Suppose that the process starts from $x \in J \cap D$. Then there will occur some excursions from $K$.

If the process $X$ hits $M_{k}$ before hitting $D^{c}$, then the part $A$ of the last excursion from $K$ before hitting $M_{k}$ is contained in $H$. Suppose without loss of generality that that excursion starts from $J$. The symmetric image of $A$ with respect to $J$ is contained in $\widetilde{H} \subset D$, by the previous step. Since the Brownian excursion law from a plane is symmetric, the process has at least the same chance of hitting $Q_{k}$ before hitting $D^{c}$, as the chance of hitting $M_{k}$ before hitting $D^{c}$ (assuming it starts from $x \in J \cap D$ ).

Step 6. It is straightforward to check that for $x \in Q_{k},|x|$ small, we have $x^{1}>2^{-k} / 40, x^{3}>2^{-k} / 40$, and $\left|x^{2}\right|<f\left(x^{1}, x^{3}\right) / 2$. Hence (4.3) and the Harnack principle yield

$$
h(x) \geq c_{12} h\left(z_{k}\right) k^{-c_{9} / a}
$$


for $x \in Q_{k}$ and large $k$. This, the previous step and (4.4) imply for $x \in J \cap D$,

$$
\begin{aligned}
\frac{1}{h(x)} \int h(y) P^{x}\left(X\left(T\left(M_{k}\right)\right) \in d y, T\left(M_{k}\right)<T\left(D^{c}\right)\right) \\
\quad \leq \frac{\int h(y) P^{x}\left(X\left(T\left(M_{k}\right)\right) \in d y, T\left(M_{k}\right)<T\left(D^{c}\right)\right)}{\int h(y) P^{x}\left(X\left(T\left(Q_{k}\right)\right) \in d y, T\left(Q_{k}\right)<T\left(D^{c}\right)\right)} \\
\quad \leq \frac{k^{-c_{9} / a-2} c_{5} h\left(z_{k}\right) P^{x}\left(T\left(M_{k}\right)<T\left(D^{c}\right)\right)}{k^{-c_{9} / a} c_{12} h\left(z_{k}\right) P^{x}\left(T\left(Q_{k}\right)<T\left(D^{c}\right)\right)} \\
\quad \leq c_{13} k^{-2} .
\end{aligned}
$$

Step 7. Now suppose that the Euclidean and Martin compactifications of $D$ are identical. We will show that this assumption leads to a contradiction. The reader may consult [Do, 1 XII 12] for the definition of the minimal fine topology.

Let $g$ be the unique (up to a multiplicative constant) minimal harmonic function in $D$ corresponding to $0 \in \partial D$. Suppose that $\left\{Y_{t}, t \geq 0\right\}$ is a $g$ process in $D$ starting from $x_{0}$. The lifetime $R$ of $Y$ is finite by the results of [BB3]. The time-reversed process $Z_{t} \stackrel{\mathrm{df}}{=} Y_{R-t}$ is an $h$-process in $D$ starting from $0 \in \partial D$ [Do, 3 III 2]. Let

$$
v(x)=\int h(y) P^{x}\left(X\left(T\left(M_{k}\right)\right) \in d y, T\left(M_{k}\right)<T\left(D^{c}\right)\right) .
$$

Then, according to (4.5),

$$
\frac{v(x)}{G_{D}\left(x, x_{0}\right)} \leq c_{13} k^{-2},
$$

for $x \in J \cap D$. By Theorem 1 XII 14 of [Do], the minimal fine limit of $v(x) / G_{D}\left(x, x_{0}\right)$ exists at $0 \in \partial D$ and is less than or equal to $c_{13} k^{-2}$. The chance that the process $Z$ ever hits $M_{k}$ is less than or equal to

$$
E\left[P_{h}^{Z_{t}}\left(T\left(M_{k}\right)<\infty\right)\right]=E\left[v\left(Z_{t}\right) / G_{D}\left(Z_{t}, x_{0}\right)\right]
$$

for every $t>0$, by the Markov property, where $E$ denotes expectation for the $Z$ process starting at 0 . The probabilistic interpretation of the minimal fine topology implies that as $t \rightarrow 0$, the integrand in the last expectation converges to a limit which does not exceed $c_{13} k^{-2}$. It follows that the probability of hitting $M_{k}$ by $Z$ is not greater than $c_{13} k^{-2}$. Since $\sum c_{13} k^{-2}<\infty$, only finitely many of the sets $M_{k}$ are hit by $Z$. Hence $Y$ approaches 0 from one side of the plane $\left\{x: x^{1}=0\right\}$. Both sides are equally probable, by symmetry, so the event that $Y$ stays eventually in $\left\{x: x^{1}>0\right\}$ has probability $1 / 2$. But this event belongs to the tail $\sigma$-field of $Y$ and, therefore, it must have probability 0 or 1 [Do, $2 \mathrm{X} 11(\mathrm{cl})$ ]. This is a contradiction which shows that the Euclidean and Martin compactifications of $D$ are different.

\section{REFERENCES}

[A] A. Ancona, Negatively curved manifolds, elliptic operators, and the Martin boundary, Ann. of Math. 125 (1987), 495-535.

[Bñ] R. Bañuelos, Intrinsic ultracontractivity and eigenvalue estimates for Schrödinger operators, J. Funct. Anal. 100 (1991), 181-206.

[BBB] R. Bañuelos, R. F. Bass and K. Burdzy, Hölder domains and the boundary Harnack principle, Duke Math. J. 64 (1991), 195-200. 
[BB1] R. F. Bass and K. Burdzy, A probabilistic proof of the boundary Harnack principle, Seminar on Stochastic Processes, 1989 (E. Cinlar, K. L. Chung and R. K. Getoor, eds.), Birkhäuser, Boston, Mass., 1990, pp. 1-16.

[BB2] _ A boundary Harnack principle for twisted Hölder domains, Ann. of Math. 134 (1991), 253-276.

[BB3] _ Lifetimes of conditioned diffusions, Probab. Theory Related Fields 91 (1992), 405-444.

[BH] R. F. Bass and P. Hsu, Some potential theory for reflecting Brownian motion in Hölder and Lipschitz domains, Ann. Probab. 19 (1991), 486-508.

[Bu] K. Burdzy, Multidimensional Brownian excursions and potential theory, Longman, New York, 1987.

[Do] J. L. Doob, Classical potential theory and its probabilistic counterpart, Springer, New York, 1984.

[FS] E. B. Fabes and D. W. Stroock, A new proof of Moser's parabolic Harnack inequality using the old ideas of Nash, Arch. Rational Mech. Anal. 96 (1986), 327-338.

[HW] R. A. Hunt and R. L. Wheeden, Positive harmonic functions on Lipschitz domains, Trans. Amer. Math. Soc. 132 (1970), 507-527.

[JK1] D. S. Jerison and C. E. Kenig, Boundary value problems on Lipschitz domains, Studies in Partial Differential Equations (W. Littman, ed.), Math. Assoc. Amer., Washington, D.C., 1982.

[JK2] - The Dirichlet problem in non-smooth domains, Ann. of Math. 113 (1981), 367-382.

[JK3] B Boundary behavior of harmonic functions in non-tangentially accessible domains, Adv. in Math. 46 (1982), 80-147.

[M] R. S. Martin, Minimal positive harmonic functions, Trans. Amer. Math. Soc. 49 (1941), 137-172.

[PS] S. C. Port and C. J. Stone, Brownian motion and classical potential theory, Academic Press, New York, 1978.

[S] M. Sharpe, General theory of Markov processes, Academic Press, Boston, Mass., 1988.

Department of Mathematics, University of Washington, Seattle, Washington 98195

E-mail address: bass@math.washington.edu

E-mail address: burdzy@math.washington.edu 